Check for updates

Cite this: Phys. Chem. Chem. Phys., 2018, 20, 17705

Received 16th May 2018, Accepted 18th June 2018

DOI: $10.1039 / \mathrm{c} 8 \mathrm{cp} 03112 f$

rsc.li/pccp

\section{Bicycloaromaticity and Baird-type bicycloaromaticity of dithienothiophene-bridged [34]octaphyrins $\dagger$}

\author{
Rashid R. Valiev, (D) ${ }^{\text {ab }}$ Heike Fliegl (iD ${ }^{c}$ and Dage Sundholm (D) *ad
}

Aromatic properties of two recently synthesized dithienothiophene-bridged (DTT) [34]octaphyrins have been investigated by calculating magnetically induced current densities and vertical excitation energies. These intriguing molecules have been proposed to be the first synthesized neutral bicycloaromatic compounds. The triplet state of their dications was even suggested to be Baird-type bicycloaromatic rendering them very interesting as a new prototype of molecules possessing simultaneously the two rare types of aromaticity. Here, we investigate computationally the aromatic properties of the neutral as well as the singly and doubly charged DTT-bridged [34]octaphyrins. Our study provides unambiguous information about changes in the aromatic properties of the DTT-bridged [34]octaphyrins upon oxidation. The calculations identify two independent diatropic ring currents in the neutral DTT-bridged [34] octaphyrins, showing that they are indeed bicycloaromatic. The current-density flow of the two independent ring currents of the bicycloaromatic compounds are visualized and individual aromatic pathways are quantified by performing numerical integration. The calculations show that two independent diatropic ring currents can indeed be sustained by molecules consisting of two aromatic rings that share a common set of $\pi$ electrons. The current density calculations on the singly charged DTT-bridged [34] octaphyrins show that they are weakly antiaromatic, which does not agree with the suggested aromatic character deduced from spectroscopical studies. The triplet state of the two DTT-bridged [34]octaphyrin cations with very similar molecular structures have unexpectedly different aromatic character. One of them is Baird-type bicycloaromatic, whereas the triplet state of the other dication has one aromatic and one nonaromatic ring, which could not be resolved from available spectroscopical data. Calculations of excitation energies reveal that a simple model cannot be employed for interpreting the electronic excitation spectra of the present molecules, because more than 20 excited states contribute to the spectra above $2.5 \mathrm{eV}(500 \mathrm{~nm})$ showing the importance of computations. The present work illustrates how detailed information about molecular aromaticity can nowadays be obtained by scrutinizing calculated current densities.

\section{Introduction}

The aromaticity concept of planar conjugated molecules fulfilling the $[4 n+2] \pi$-electron count rule is well known. ${ }^{1,2}$ Even though aromaticity is still not completely understood, it is an important concept in chemistry. It has constantly been a topic of debate and thoroughly investigated using different kinds

\footnotetext{
${ }^{a}$ Department of Chemistry, University of Helsinki, P.O. Box 55, FIN-00014 Helsinki, Finland. E-mail: Dage.Sundholm@helsinki.fi

${ }^{b}$ Department of Optics and Spectroscopy, Tomsk State University, Lenina Avenue 36, Tomsk, Russian Federation ${ }^{c}$ Hylleraas Centre for Quantum Molecular Sciences, Department of Chemistry, University of Oslo, P.O. Box 1033, Blindern, 0315 Oslo, Norway

${ }^{d}$ Centre for Advanced Study at the Norwegian Academy of Science and Letters, Drammensveien 78, N-0271 Oslo, Norway

$\dagger$ Electronic supplementary information (ESI) available: The Cartesian coordinates of the investigated molecules. Discussion of strengths of the current pathways and plots of the modulus of the current densities are reported. See DOI: 10.1039/c8cp03112f
}

of experimental and computational approaches. ${ }^{3,4}$ Various theoretical methods have been employed in order to develop reliable aromaticity indices for comparisons of the degree of molecular aromaticity. ${ }^{3}$ We recently showed that the degree of aromaticity obtained using the magnetic ring-current and the energetic criteria have a linear correlation. ${ }^{5}$ Thus, the magnetic ring-current criterion can be used for assessing the degree of aromaticity in a broader sense. Ring-current strengths are indirectly explored in the popular nucleus independent chemical shift (NICS $)^{6-8}$ method and in calculations of the anisotropy of the induced current density (ACID). ${ }^{9-11}$ However, the NICS and ACID approaches are not able in all cases to accurately predict the aromatic character for complicated molecular ring systems. ${ }^{11-18}$ Instead calculations of magnetically induced current densities provide an unambiguous picture of the current flow. Integration of the current strength passing selected planes yields ring-current strengths and current pathways in the molecule. Interpretation of the huge amount of the current-density information is though 
challenging especially when aiming at a very detailed understanding of the aromatic properties. ${ }^{14,19,20}$ Combination of numerical integration of the current flow and advanced visualization methods provide an accurate picture of the magnetically induced current density in complicated molecules.

Expanded porphyrins are well suited for combined experimental and theoretical studies of molecular aromaticity, because many different kinds of expanded porphyrins have been synthesized and systematically modified for investigating different aspects of aromaticity. ${ }^{4,21-24}$ It is even possible to synthesize nonplanar Möbius twisted porphyrinoid structures, whose aromatic properties have been studied experimentally and computationally. ${ }^{25-32}$ Studies show that porphyrins with $[4 n+1] \pi$ electrons can be stable similar to aromatic molecules with $[4 n+2] \pi$ electrons. $^{33-38}$

Aromaticity rules have been extended to molecules in triplet state. Baird's aromaticity rule states that molecules in triplet state are aromatic when they have [4n] $\pi$-electrons, while those with $[4 n+2] \pi$ electrons are antiaromatic..$^{39}$ Baird-type aromaticity is presently an active research topic, because it provides important information about excited-state aromaticity that is of importance for photophysical processes of molecules. ${ }^{29,40-44}$

Bicycloaromaticity is an aromaticity concept that was originally suggested by Goldstein and Hoffmann. ${ }^{45,46}$ The underlying idea is that in nonplanar molecules two aromatic $\pi$-electron circuits can exist independently of each other. Two connected molecular rings with a common conjugation pathway in one part of them share $\pi$ electrons that sustain independent ring currents. The basic bicycloaromaticity idea was proposed in 1967, whereas it lasted 50 years until the first neutral bicycloaromatic molecules were synthesized. ${ }^{33,47,48}$ Cha et al. recently reported synthesis and spectroscopic characterization of two dithienothiophene (DTT)bridged [34]octaphyrins. Measurements of nuclear magnetic resonance (NMR) chemical shifts suggested that the synthesized molecules are bicycloaromatic with two connected conjugation loops with 26 and $34 \pi$ electrons that sustain two independent ring currents. They proposed that two electron oxidation of the bicycloaromatic molecules leads to a triplet state with $[4 n+1] \pi$ electrons in each loop. The studies suggested that the DTT-bridged [34]octaphyrins are the first example of neutral bicycloaromatic molecules and that the dications are the first example of molecules with Baird-type bicycloaromaticity. ${ }^{33}$ They also proposed that one electron oxidation leads to different aromatic properties for the cations of the two synthesized DTT-bridged [34]octaphyrins. It was also suggested that in one case the oxidation resulted in a globally aromatic cation, where half of an electron was removed from both loops, whereas for the other molecule one electron was removed from the larger loop and the smaller ring has the same number of $\pi$ electrons as the corresponding neutral molecule. ${ }^{33}$

Cha et al. investigated the aromatic properties of the neutral molecules spectroscopically using ${ }^{1} \mathrm{H}$ NMR spectroscopy and ultraviolet-visible (UV-VIS)-near-infrared (NIR) absorption spectroscopy, whereas for the charged molecules they did not report any ${ }^{1} \mathrm{H}$ NMR chemical shifts. Thus, the aromatic character of the charged molecules is based only on changes in the UV-VIS-NIR spectra upon oxidation. ${ }^{33}$ The spin state of the charged species was determined by measuring the electron paramagnetic resonance
(EPR) spectra. The spectroscopical characterization was supported by calculations of nucleus independent chemical shifts (NICS) ${ }^{8}$ and anisotropy of the induced current density (ACID) plots. ${ }^{49}$

Here, we investigate the aromatic properties of the DTT-bridged [34]octaphyrins by calculating magnetically induced current densities using our gauge including magnetically induced current (GIMIC) method. ${ }^{19,50-52}$ The pathways of the magnetically induced ring currents have been obtained by integrating the current flow passing selected chemical bonds of the molecular rings. The ring-current strengths are used for determining the degree of aromaticity, whereas the direction of the ring current i.e., the tropicity shows whether the rings are aromatic or antiaromatic. The UV-VIS-NIR spectra have been calculated at the expanded multi-configurational quasi-degenerate perturbation theory level.

\section{Computational methods}

The molecular structures of all the studied molecules were optimized at the density functional theory (DFT) level using the B3LYP functional and the def2-TZVP basis set with Turbomole version 7.2. ${ }^{53-57}$ The molecular structure of the triplet state of $1 \mathrm{a}^{2+}$ was also optimized using the M06-2X functional. ${ }^{58}$ Nuclear magnetic resonance (NMR) shielding constants were calculated at the DFT level with the B3LYP, BHLYP and M06-2X functionals ${ }^{53,54,59}$ and def2-TZVP basis set ${ }^{60}$ using the mpshift ${ }^{61,62}$ module of Turbomole for the closed-shell molecules and Gaussian $09^{63}$ for the open-shell species.

Gauge-including atomic orbitals (GIAO) were used in the NMR shielding calculations. ${ }^{64,65}$ Magnetically induced current densities were obtained using the gauge including magnetically induced current method (GIMIC). ${ }^{19,50-52}$ GIMIC is interfaced to Turbomole and Gaussian 09. ${ }^{50,66}$ GIMIC uses basis set information, the atomic orbital density matrix and the perturbed atomic orbital density matrices as input data, which are obtained by performing. NMR shielding calculations. In the current-density calculations, the nonplanar molecules were oriented largely in the $x y$ plane with the magnetic field in $z$ direction. The BHLYP functional ${ }^{59}$ with $50 \%$ Hartree-Fock (HF) exchange has been found to be a better choice than B3LYP for calculating magnetically induced current densities of antiaromatic molecules, because BHLYP calculations yield similar current densities as obtained at the second-order Møller-Plesset perturbation theory (MP2) level. ${ }^{24}$ The B3LYP functional tends to overestimate the paratropic ring-current strengths of strongly antiaromatic molecules. ${ }^{67}$ However, the B3LYP functional yields accurate ring-current strengths for aromatic molecules that are dominated by diatropic current densities. The magnetically induced current density for the triplet state of $\mathbf{1 a}^{2+}$ was also calculated with a new version of the mpshift program using the M06-2X functional, which has $54 \% \mathrm{HF}$ exchange, and the molecular structure optimized at the M06-2X level. ${ }^{58,62}$ The differences between the molecular structures optimized at the B3LYP and M06-2X levels are very small, which does not support the notion that the B3LYP functional has difficulties to describe the electronic structure of porphyrinoids. ${ }^{68}$ Current density calculations show that aromatic porphyrinoids are well described 
at the DFT level using common functionals, whereas the strength of the paratropic ring current of porphyrinoids decreases when increasing the amount of HF exchange. ${ }^{24,67}$ Functionals with about $50 \% \mathrm{HF}$ exchange yield ring-current strengths of about the same strength as obtained at the MP2 level. ${ }^{24}$

A recent overview of current density studies on porphyrinoids is given in ref. 20. For the visualization of the streamlines of the three-dimensional current density, the corresponding Paraview plugin has been employed using a radius of 0.4 bohr for the inspection sphere. ${ }^{69}$ The molecular structures have been visualized with $\mathrm{VMD}^{70}$ and ChemCraft. ${ }^{71}$

Excitation energies with the same spin state as the ground state were calculated at the expanded multi-configurational quasi-degenerate perturbation theory (XMC-QDPT2) level using the def2-TZVP basis sets. ${ }^{60,72}$ The complete active space selfconsistent field (CASSCF) calculations considered 6 electrons in 6 molecular orbitals. We performed state-average CASSCF (SA-CASSCF) calculations on the 1a and 2a species using 21 states for the neutral form, 26 states for the cation, and 28 states for the dication. In the XMC-QDPT2 calculations, the effective Hamiltonian comprised 35 states for the neutral molecules, 45 states for the cations, and 50 states for the dications. The oscillator strengths were also calculated at the XMC-QDPT2 level. The excitation energies and oscillator strengths were calculated with the Firefly software. ${ }^{73}$

\section{Results and discussion}

\subsection{Molecular structures}

The molecular structures of the DTT-bridged [34] octaphyrins 1a and $\mathbf{2 a}$ as well as their planar [26]hexaphyrin-like building blocks $\mathbf{1 b}$ and $\mathbf{2 b}$ are shown in Fig. $\mathbf{1}$. We use the same notation

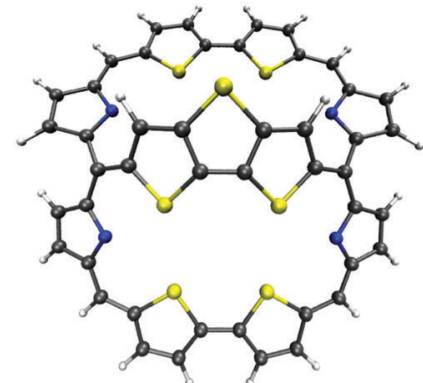

(1a)

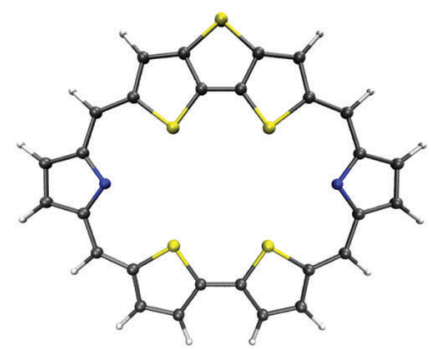

(1b)

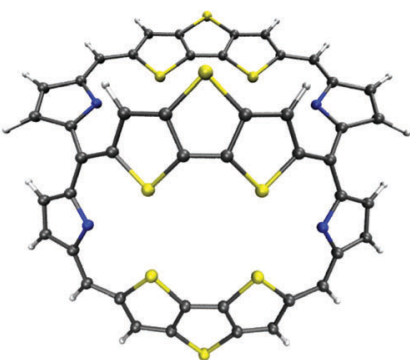

(2a)

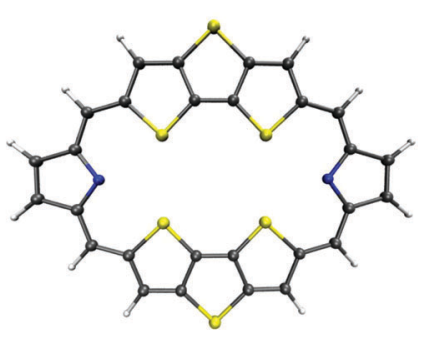

(2b)
Fig. 1 The molecular structures of the investigated DTT-bridged [34]octaphyrins $\mathbf{1 a}$ and $\mathbf{2 a}$, and their [26] hexaphyrin like building blocks $\mathbf{1 b}$ and $\mathbf{2 b}$. as introduced by Cha et al. ${ }^{33}$ The structural difference between 1a and $\mathbf{2 a}$ is that in 1a, the two thiophene rings are connected by one bond, whereas in 2a the thiophene rings are annelated via an additional sulfur bridge. Molecules $\mathbf{1 b}$ and $\mathbf{2 b}$ have the same structural differences as the smaller molecular ring in 1a and 2a. The DTT-bridged [34] octaphyrins are slightly nonplanar. The Cartesian coordinates of the molecular structures are given in the ESI. $†$

\subsection{Current pathways in $1 \mathrm{a}$ and $2 \mathrm{a}$}

The 3D streamline visualization of the current densities of $\mathbf{1 a}$ and $2 \mathrm{a}$ in Fig. 2 shows that the molecules sustain two spatially independent ring currents. The blue streamlines were obtained by placing the inspection sphere of the plotting algorithm $1 \mathrm{bohr}$ above the carbon atom fusing both rings and the red one was obtained with the sphere placed 1 bohr below the carbon atom. The two current-density flows are almost planar. The plane of the ring current of the small ring shown in blue is slightly tilted with respect to the plane of the ring current of the large loop shown in red. The streamline plots suggest that molecules $\mathbf{1 a}$ and $\mathbf{2 a}$ are indeed bicycloaromatic, which was also found experimentally by Cha et al. ${ }^{33}$

Numerical integration of the current strengths passing selected chemical bonds shows that molecules 1a and 2a sustain similar current pathways and current strengths. The current strengths of 1a are followed by the ones for $\mathbf{2 a}$ within parenthesis. The small loop

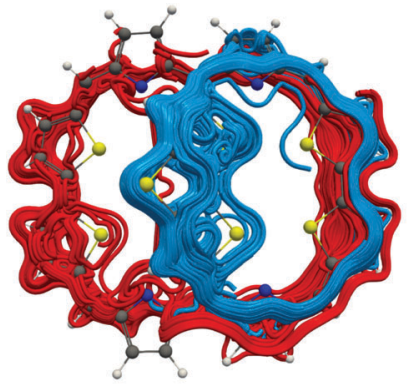

(a)

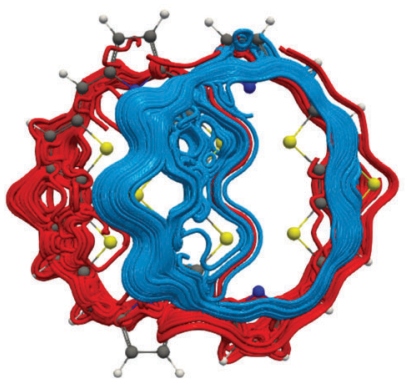

(c)

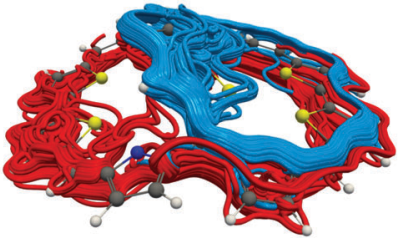

(b)

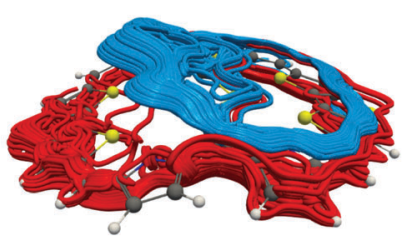

(d)
Fig. 2 The magnetically induced ring currents of the two rings of 1a are shown in ( $a$ and $b$ ). Figures ( $c$ and d) show the corresponding ring currents of $2 \mathrm{a}$. The visualization has been done with the 3D streamline visualization plugin of ParaView using a sphere radius of 0.4 bohr. For streamlines depicted in blue, the inspection sphere was placed 1 bohr above the fusing carbon atom at the DDT bridge, while for the red streamlines the sphere was placed 1 bohr below the neighboring carbon atom to the left of the junction. 


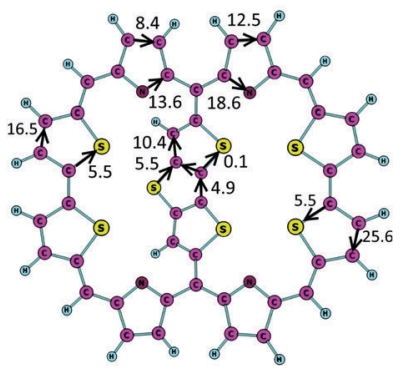

(1a)

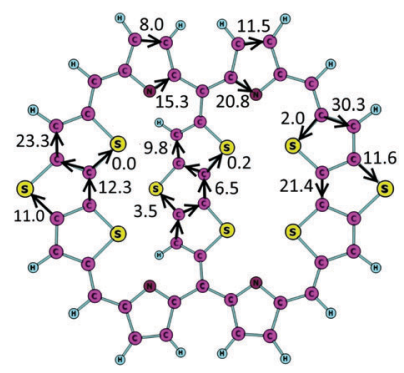

(2a)
Fig. 3 The strengths (in $n A T^{-1}$ ) of the current pathways of $1 \mathbf{a}$ and $2 a$ calculated at the B3LYP/def2-TZVP level of theory.

in 1a sustains a ring current of $10.5 \mathrm{nA} \mathrm{T}^{-1}\left(10.0 \mathrm{nA} \mathrm{T}^{-1}\right)$. The current strength of the large loop is $22.0 \mathrm{nA} \mathrm{T}^{-1}\left(23.3 \mathrm{nA} \mathrm{T}^{-1}\right)$ and $10.5 \mathrm{nA} \mathrm{T}^{-1}\left(10.0 \mathrm{nA} \mathrm{T}^{-1}\right)$ passes the bridge. The calculated current pathways and current strengths are shown in Fig. 3. A detailed description of the current pathways and their current strengths is given in the ESI. $\dagger$

\subsection{Current pathways in the triplet state of $1 \mathrm{a}^{2+}$ and $2 \mathrm{a}^{2+}$}

Calculation of the spin $\alpha$ and spin $\beta$ contributions to the current density of $\mathbf{1 a}^{2+}$ shows that the spin $\beta$ contribution to the current density is paratropic in the large molecular ring and that the spin $\alpha$ contribution to the current density is diatropic in both molecular rings leading to a nonaromatic character of the large molecular ring and an aromatic character for the small molecular ring. Since the number of $\beta$ electrons is two fewer that the number of $\alpha$ electrons, the oxidation removed two electrons from the large molecular ring according to the present calculations. The $3 \mathrm{D}$ streamline visualization of the current density and the spin-current densities of $\mathbf{1 a ^ { 2 + }}$ are shown in Fig. 4. Modulus plots of the spin current densities of the studied molecular systems are given in the ESI. $\dagger$

Calculation of the spin $\alpha$ and spin $\beta$ contributions to the current density for $2 \mathrm{a}^{2+}$ shows that both spin currents are diatropic in the two molecular rings leading to the Baird-type bicycloaromatic character of the triplet state of $2 \mathbf{a}^{2+}$. The current density calculation suggests that one electron is removed from the large molecular ring and one from the small one when doubly oxidizing $2 a$. Thus, the oxidation does not lead to any significant changes in the aromatic character in this case. The 3D streamline visualization of the current density and the spin-current densities of $2 \mathbf{a}^{2+}$ are shown in Fig. 5.

The current strength and current pathways of the triplet state of $\mathbf{1 a}^{2+}$ differ significantly from those of $\mathbf{1 a}$. The large loop sustains a very weak ring current of $3.2 \mathrm{nA} \mathrm{T}^{-1}$ as compared to $22.0 \mathrm{nA} \mathrm{T}^{-1}$ for 1a. The current strength of the small loop is $29.0 \mathrm{nA} \mathrm{T} \mathrm{T}^{-1}$ for $\mathbf{1 a}^{2+}$, which is almost three times stronger than for 1a. Thus, the small loop is strongly aromatic, whereas the large loop of the doubly charge $\mathbf{1 a}^{2+}$ is practically nonaromatic with aromatic thiophene rings. The pyrrolic rings of the large loop are almost nonaromatic.

Calculations of the current density at the M06-2X level yielded practically the same current pathway and current strengths

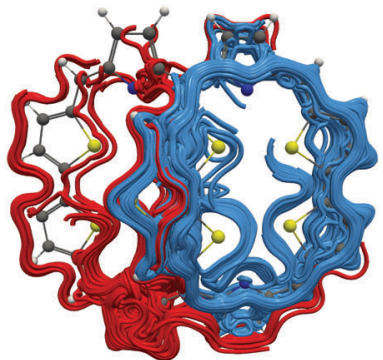

(a)

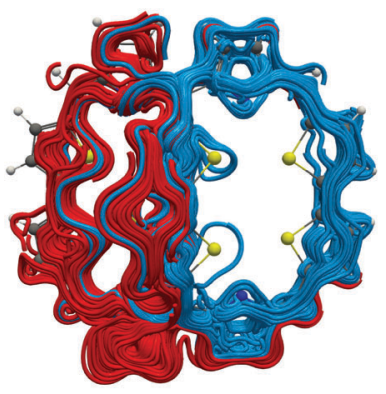

(c)

Fig. 4 Calculated current density represented as 3D streamlines for (a) the total current density, (b) the $\alpha$ contribution and (c) $\beta$ contribution to the total current density of $1 \mathrm{a}^{2+}$. For streamlines depicted in blue, the inspection sphere was placed 1 bohr above the fusing carbon atom at the DDT bridge, while for the red streamlines the sphere was placed 1 bohr below the neighboring carbon atom to the left of the junction.

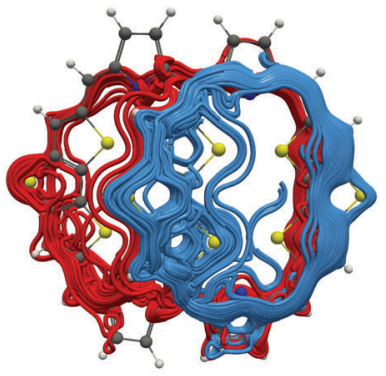

(a)

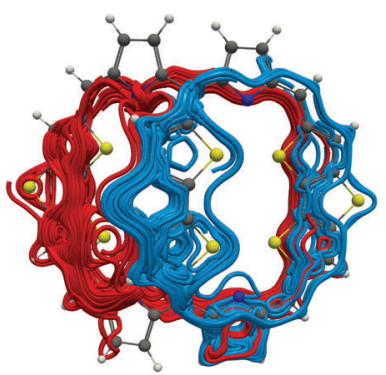

(c)

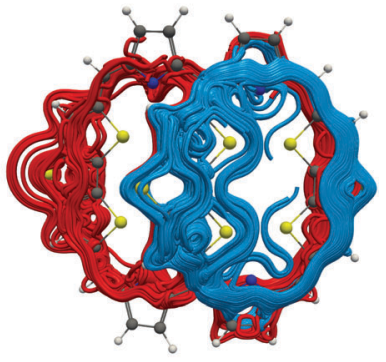

(b)
Fig. 5 The 3D streamlines for (a) the total current density, (b) the $\alpha$ contribution and (c) $\beta$ contribution to the total current density of $2 a^{2+}$. The streamlines depicted in blue was obtained with the inspection sphere 1 bohr above the fusing carbon atom at the DDT bridge, while for the red streamlines the sphere was placed 1 bohr below the neighboring carbon atom to the left of the junction.

for the large loop as obtained in the B3LYP calculation. At the M06-2X level, the current strength of the small loop is $9 \mathrm{nA} \mathrm{T}$ 


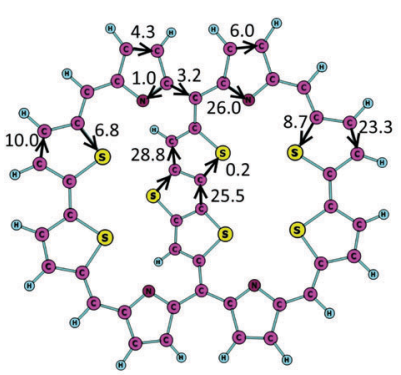

$\left(1 \mathrm{a}^{2+}\right)$

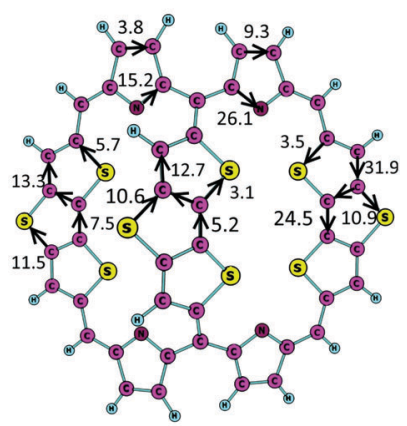

$\left(2 \mathrm{a}^{2+}\right)$
Fig. 6 The strengths (in $\mathrm{nA} \mathrm{T}^{-1}$ ) of the current pathways of the triplet state of the $1 a^{2+}$ and $2 a^{2+}$ dications calculated at the B3LYP/def2-TZVP level of theory.

weaker than the one obtained in the B3LYP calculation. However, the current pathways are qualitatively the same at both levels of theory showing that the small loop of the triplet state of $1 \mathbf{a}^{2+}$ is aromatic and the large loop is almost nonaromatic.

The current strengths of $2 \mathrm{a}^{2+}$ followed by the ones for neutral $2 \mathrm{a}$ within parenthesis. The current pathways of the triplet state of the doubly charged $2 \mathrm{a}^{2+}$ are similar to those of the neutral $2 \mathrm{a}$. The small loop in $2 \mathrm{a}^{2+}$ sustains a ring current of $15.8 \mathrm{nA} \mathrm{T}^{-1}\left(10.0 \mathrm{nA} \mathrm{T}^{-1}\right)$ and for the large loop is it is $19.0 \mathrm{nA} \mathrm{T}^{-1}\left(23.3 \mathrm{nA} \mathrm{T}^{-1}\right)$. The calculated current strengths along the different pathways for the triplet state of $1 \mathrm{a}^{2+}$ and $2 \mathrm{a}^{2+}$ are shown in Fig. 6 . The current pathways and their current strengths are discussed in detail in the ESI. $\dagger$

Current density calculations on the triplet state of $\mathbf{1 a ^ { 2 + }}$ show that the small loop is aromatic, whereas the large loop sustains a weak diatropic ring current. The triplet state of $2 \mathbf{a}^{2+}$ has a completely different aromatic character, as its large and the small loops sustain strong diatropic ring currents. Thus, the triplet state of $2 \mathrm{a}^{2+}$ is Baird-type bicycloaromatic, whereas the triplet state of $\mathbf{1 a}^{2+}$ has only one aromatic macro ring. Cha et al. suggested that the triplet state of both $1 \mathrm{a}^{2+}$ and $2 \mathrm{a}^{2+}$ are Baird-type bicycloaromatic, ${ }^{33}$ which does not agree with the results obtained in the present computational study.

\subsection{Current pathways in the doublet state of $1 \mathrm{a}^{1+}$ and $2 \mathrm{a}^{1+}$}

Calculations of the spin $\alpha$ and spin $\beta$ contributions to the current density for $1 \mathbf{a}^{1+}$ and $2 \mathrm{a}^{\mathbf{1}^{+}}$show that the spin $\beta$ contribution to the current density is paratropic in the two molecular rings whereas the spin $\alpha$ contribution is diatropic but weaker than the paratropic current density contribution of the $\beta$ electrons leading to a weakly antiaromatic character of the doublet state of $1 \mathbf{a}^{1+}$ and $2 \mathbf{a}^{1+}$. The two ring currents and the spin contributions to the ring currents are visualized in Fig. 7.

The doublet state of the $1 \mathbf{a}^{1+}$ and $2 \mathbf{a}^{1+}$ cations are globally antiaromatic sustaining very similar current pathways and current strengths. The current strengths of $1 \mathrm{a}^{1+}$ are followed by the ones for $2 \mathbf{a}^{1+}$ within parenthesis. The small loop of $1 \mathbf{a}^{1+}$ sustains a paratropic ring current of $-8.0 \mathrm{nA} \mathrm{T}^{-1}\left(-11.0 \mathrm{nA} \mathrm{T}^{-1}\right)$. The current strength of the large loop is $-2.4 \mathrm{nA} \mathrm{T}^{-1}\left(-5.2 \mathrm{nA} \mathrm{T}^{-1}\right)$ and $-5.9 \mathrm{nA} \mathrm{T}^{-1}\left(-5.8 \mathrm{nA} \mathrm{T}^{-1}\right)$ passes the bridge.

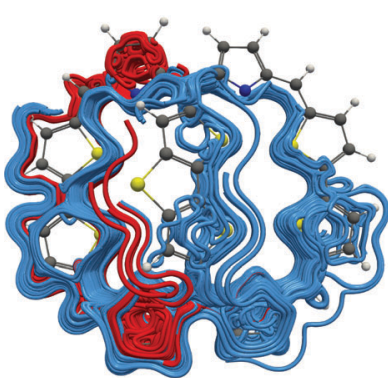

(a)

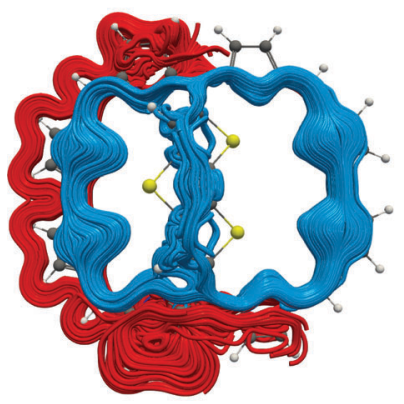

(c)

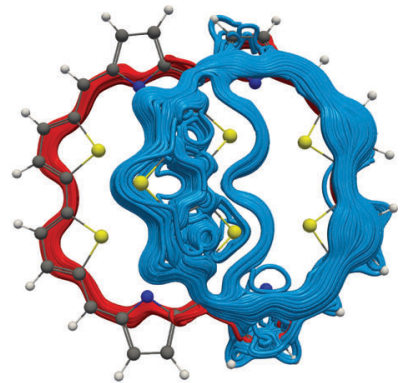

(b)
Fig. 7 Calculated current density represented as 3D streamlines for (a) the total current density, (b) the $\alpha$ contribution and (c) the $\beta$ contribution to the current density of $\mathbf{1 a}^{+}$. For streamlines depicted in blue the inspection sphere was placed 1 bohr above the fusing carbon atom at the DDT bridge, while for the red streamlines the sphere was placed 1 bohr below the neighboring carbon atom to the left of the junction.

A similar current pathway pattern is obtained for the bridges of $1 \mathbf{a}^{1+}$ and $2 \mathbf{a}^{1+}$ and for the three thiophene rings of the common part of the two loops of molecule $2 \mathbf{a}^{1+}$. The global paratropic ring current passes on the inside of the three thiophene rings. The calculated current pathways and current strengths for $1 \mathbf{a}^{1+}$ and $\mathbf{2 \mathbf { a } ^ { 1 + }}$ are shown in Fig. 8. The current pathways are discussed more thoroughly in the ESI. $\dagger$

The aromatic properties for the $1 \mathbf{a}^{1+}$ and $2 \mathbf{a}^{1+}$ cations differ completely from those suggested in the experimental work by Cha et al. ${ }^{33}$ In the experimental characterization they suggested that $1 \mathrm{a}^{\mathbf{1}^{+}}$is globally aromatic with one electron missing in the large loop and that the aromatic properties of the small loop are the same as for the corresponding neutral molecule. For $2 \mathbf{a}^{1+}$, they suggested that the global aromaticity is weakened,

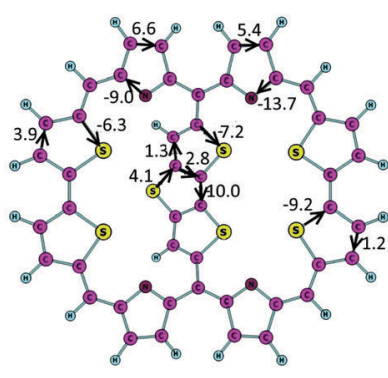

$\left(1 a^{1+}\right)$

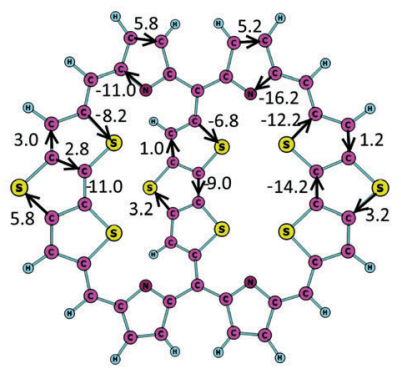

$\left(2 a^{1+}\right)$
Fig. 8 The strengths (in $n A T^{-1}$ ) of the current pathways of $1 a^{1+}$ and $2 a^{1+}$ calculated at the B3LYP/def2-TZVP level of theory. 
because half an electron is removed from both loops. The conclusions were drawn based on changes in the measured UV-VIS-NIR spectra upon oxidation. ${ }^{33}$ The present calculations suggest on the other hand that the large loop of $1 \mathbf{a}^{1+}$ is very weakly antiaromatic and $2 \mathbf{a}^{1+}$ sustains a somewhat stronger paratropic ring current in the large loop. The current pathways and current strengths for the rest of the two cations are similar. The main conclusion of the present calculations is that paratropic current densities dominate in $\mathbf{1 a}^{1+}$ and $2 \mathbf{a}^{1+}$, which is not in agreement with the conclusion drawn in the experimental study by Cha et ll. $^{33}$

\subsection{Current pathways of the $1 \mathrm{~b}$ and $2 \mathrm{~b}$ species}

The singlet states of the neutral $\mathbf{1 b}$ and $\mathbf{2 b}$ rings are aromatic sustaining a ring current strength of $34.7 \mathrm{nA} \mathrm{T}^{-1}$ and $34.0 \mathrm{nA} \mathrm{T}^{-1}$, respectively. Thus, $\mathbf{1 b}$ and $\mathbf{2 b}$ are strongly aromatic as also obtained in the experimental study. ${ }^{33}$ The current pathways and current strengths of $\mathbf{1 b}$ and $\mathbf{2 b}$ discussed in the ESI $\dagger$ are also practically the same.

The triplet states of the $\mathbf{1} \mathbf{b}^{2+}$ and $\mathbf{2} \mathbf{b}^{2+}$ dications are aromatic sustaining a ring current strength of $31.2 \mathrm{nA} \mathrm{T}^{-1}$ and $33.7 \mathrm{nA} \mathrm{T}^{-1}$, respectively. The triplet state of $\mathbf{1} \mathbf{b}^{2+}$ and $2 \mathbf{b}^{2+}$ are Baird-type aromatic, as they also sustain very strong diatropic ring currents that are of the same strength as for neutral $\mathbf{1 b}$ and $\mathbf{2 b}$. The current strengths and pathways are more thoroughly discussed in the ESI. $\dagger$

The singlet state of the $\mathbf{1} \mathbf{b}^{2+}$ dication is strongly antiaromatic sustaining a net paratropic ring current of $-78.4 \mathrm{nA} \mathrm{T}^{-1}$ at the B3LYP level. However, the B3LYP functional has a tendency to overestimate the degree of antiaromaticity for strongly antiaromatic molecules, ${ }^{24,67}$ whereas for aromatic porphyrinoids about the same ring-current strengths are obtained at the MP2 level and at the DFT level using different functionals. ${ }^{67}$ The current strengths calculated at the B3LYP level for strongly antiaromatic porphyrinoids were found to be about a factor of 2-3 larger than the current strengths obtained at the MP2 level, whereas the BHLYP functional yielded ring-current strengths that agreed well with the MP2 ones. ${ }^{67}$ The ring-current strength for the singlet state of the $\mathbf{1 b}^{2+}$ dication calculated using the BHLYP functional is $-41.0 \mathrm{nA} \mathrm{T}^{-1}$. The strengths of the current pathways calculated at the B3LYP and BHLYP levels are compared in the ESI. $\dagger$ The ring-current strength calculations show that the singlet state of $\mathbf{1} \mathbf{b}^{2+}$ is indeed strongly antiaromatic as also suggested in the experimental work. ${ }^{33}$

\section{Comparison of experimental and theoretical results}

Characterization of the recently synthesized dithienothiophenebridged [34]octaphyrins (1a and 2a) showed that the molecules with formally $60 \pi$ electrons are aromatic, because the two connected conjugation pathways with 26 and $34 \pi$ electrons, respectively, sustain independent aromatic pathways fulfilling the Hückel rule for aromaticity. ${ }^{33}$ The present calculations of the ring-current strengths and ring-current pathways support the experimental results for neutral 1a and 2a. Thus, our computational study confirms that the synthesized molecules are neutral bicycloaromatic molecules.

Experimental characterization of the aromatic properties of the corresponding charged molecules is less straightforward, because ${ }^{1} \mathrm{H}$ NMR spectra have not been recorded for them. However, measurements of the UV-Vis-NIR spectra suggested that the oxidation of $\mathbf{1 a}$ leads to an electronic structure with $4 n+1 \pi$-electrons in the large loop, whereas the number of $\pi$ electrons in the small loop is the same as for neutral 1a. The spectroscopical studies suggested that further oxidation of $\mathbf{1 a}^{1+}$ leads to a triplet ground state of $\mathbf{1 a}^{2+}$ with 33 and $25 \pi$ electrons in the large and the small loop, respectively. ${ }^{33}$ However, the conclusions drawn from the spectroscopical studies are not supported by the present calculations. The UV-Vis-NIR spectra calculated at the XMC-QDPT2 level are compared in Fig. 9. The XMC-QDPT2 calculations of the UV-Vis-NIR spectra showed that the studied molecules have more than 20 excited states

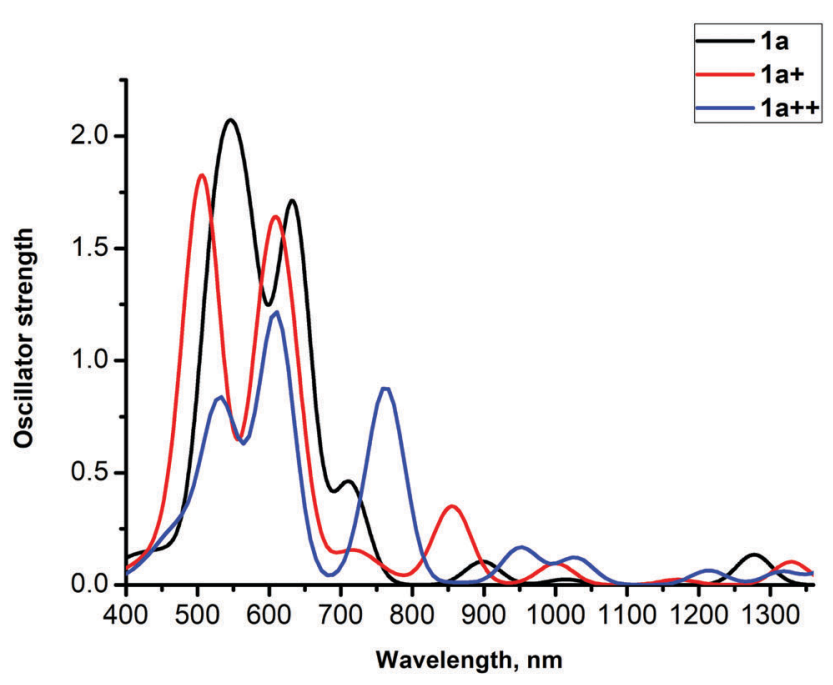

(a)

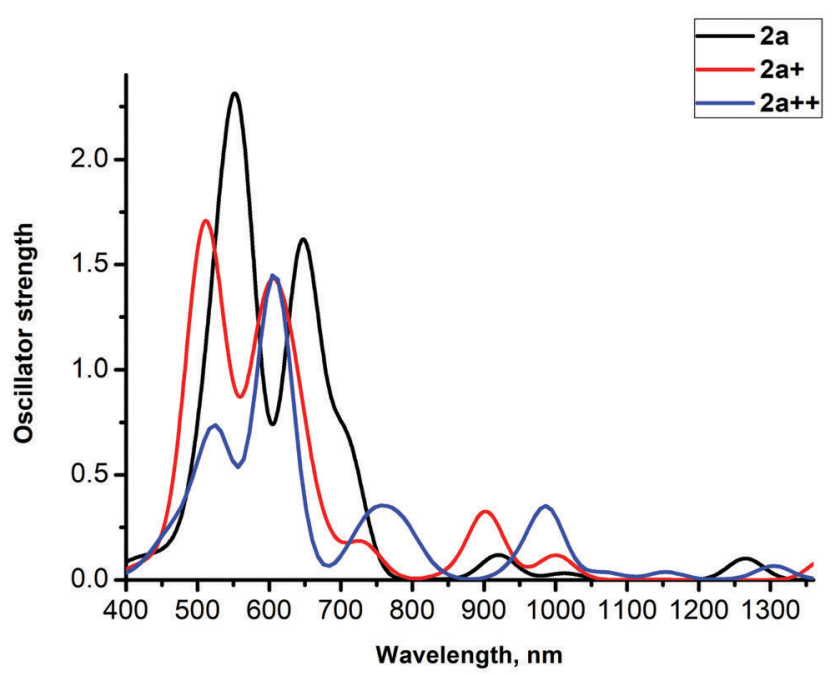

(b)

Fig. 9 Comparison of the UV-Vis-NIR spectra of (a) $1 a, 1 a^{1+}, 1 a^{2+}$, and (b) $\mathbf{2 a}, \mathbf{2} \mathbf{a}^{1+}, \mathbf{2} \mathbf{a}^{2+}$ calculated at the XMC-QDPT2 level. 
above $2.5 \mathrm{eV}$ (500 $\mathrm{nm}$ ), implying that a simple model cannot be used for interpreting the spectra.

The current-density calculations show that the small loop of $1 \mathbf{a}^{1+}$ is weakly antiaromatic and that the large loop is very weakly antiaromatic or almost nonaromatic. For $\mathbf{1 a}^{2+}$ we find that the small loop is aromatic and the large one is practically nonaromatic sustaining a very weak diatropic ring current. The present computational study does not support the notion that $\mathbf{1 a}^{2+}$ is a Baird-type bicycloaromatic molecule.

The experimental UV-Vis-NIR spectra for singly oxidized $2 \mathbf{a}^{\mathbf{1}^{+}}$ suggested that both loops are affected when removing an electron from $2 a^{33}$ Cha et al. suggested that the two loops have 25.5 and $33.5 \pi$ electrons, respectively. ${ }^{33}$ Further oxidation of $2 \mathbf{a}^{1+}$ yields $2 \mathbf{a}^{2+}$ in the triplet state with 33 and $25 \pi$ electrons in the two loops. Thus, the aromatic properties for $\mathbf{2 a ^ { 2 + }}$ that were deduced from the experimental studies were very similar to those of $1 \mathbf{a}^{2+}$. Current density calculations on $2 \mathbf{a}^{1+}$ yielded very similar ring-current strengths and current pathways as for $1 \mathbf{a}^{\mathbf{1}^{+}}$. Thus, the small loop of $2 \mathbf{a}^{1+}$ is weakly antiaromatic and the large one is very weakly antiaromatic or practically nonaromatic.

Current density calculations on $\mathbf{2 a ^ { 2 + }}$ show that the small and large rings are aromatic sustaining strong ring currents of $15.8 \mathrm{nA} \mathrm{T}^{-1}$ and $19.0 \mathrm{nA} \mathrm{T}{ }^{-1}$. The present calculations suggest that $2 \mathrm{a}^{2+}$ is indeed a Baird-type bicycloaromatic molecule.

The present study shows that it is very challenging to experimentally estimate the aromatic character of large molecules with multiple rings without having access to ${ }^{1} \mathrm{H}$ NMR spectra. In the present case, the conclusions from the computational and experimental studies agree completely for the neutral molecules that could be thoroughly characterized experimentally using ${ }^{1} \mathrm{H}$ NMR spectroscopy and other spectroscopical techniques, whereas for the charged species with lacking experimental ${ }^{1} \mathrm{H}$ NMR data, the experimental interpretation had to rely on other advanced spectroscopies that are apparently not able to reliable assess the degree of molecular aromaticity, illustrating the importance of the interplay between experimental and theoretical investigations.

\section{Summary}

Magnetically induced current densities have been calculated and analyzed for two recently synthesized dithienothiophene-bridged [34] octaphyrins (1a and 2a) and for the corresponding singly and doubly oxidized cations as well as for their hexaphyrin-type building blocks. Our computational study comprises detailed investigations of magnetically induced ring-current strengths and current-density pathways of the neutral and charged molecules. The calculations show that the two macrorings sustain strong diatropic ring currents indicating that the neutral molecules are bicycloaromatic molecules as recently suggested based on spectroscopical studies. ${ }^{33}$

We have also investigated how the current density flow changes when oxidizing the molecules. The doublet state of the singly charged cations were found to sustain paratropic ring currents indicating that $1 \mathbf{a}^{1+}$ and $2 \mathbf{a}^{1+}$ are weakly antiaromatic or almost nonaromatic, which is not in agreement with the conclusions drawn in the experimental study. ${ }^{33}$
Table 1 Comparison of the calculated net current strengths circling around the large ring, passing the common part of the large and the small ring, and the current strengths passing the bridge. All values are reported in $n A T^{-1}$

\begin{tabular}{lccr}
\hline Molecule & Large ring & Common part & Bridge \\
\hline $\mathbf{1 a}$ & 22.0 & 31.1 & 9.1 \\
$\mathbf{2 a}$ & 23.3 & 32.3 & 9.1 \\
$\mathbf{1 a}^{1+}$ & -2.4 & -8.0 & -5.6 \\
$\mathbf{2 a}^{1+}$ & -5.2 & -11.0 & -5.8 \\
$\mathbf{1 a}^{2+}$ & 3.2 & 32.0 & 28.8 \\
$\mathbf{2 \mathbf { a } ^ { 2 + }}$ & 19.0 & 35.4 & 16.4 \\
\hline
\end{tabular}

The current density calculations show that the triplet state of $1 \mathbf{a}^{2+}$ and $2 \mathbf{a}^{2+}$ have different aromatic characters. The small molecular ring is aromatic in $\mathbf{1} \mathbf{a}^{2+}$, whereas the large loop is practically nonaromatic. The triplet state of $2 \mathbf{a}^{2+}$ sustaining diatropic ring currents in both the large and the small loop is on the other hand a Baird-type bicycloaromatic molecule. The calculated net current strength susceptibilities of the main rings are summarized in Table 1.

Current density calculations on the hexaphyrin building blocks show that the neutral molecules are aromatic. The doubly charged cation in the singlet state is antiaromatic and the triplet state of the doubly charged cations is aromatic. Thus, the hexaphyrin rings of $\mathbf{2 a}$ and $2 \mathbf{a}^{2+}$ fulfil the Hückel and Baird rules for aromaticity, respectively.

The studied molecules show very interesting aromatic properties that are difficult to determine experimentally when ${ }^{1} \mathrm{H}$ NMR spectroscopical measurements are not available. Aromatic properties deduced from experimental UV-VIS-NIR spectroscopy cannot be trusted because the studied molecules have more than 20 excited states in the NIR-Vis part of the spectrum implying that simple empirical rules can not be used for interpreting the optical spectra.

\section{Conflicts of interest}

There are no conflicts to declare.

\section{Acknowledgements}

The Academy of Finland has supported this work through projects 275845 and 297304. H. F. thanks the Norwegian Research Council through the Hylleraas Centre for Quantum Molecular Sciences (Grant No. 262695 and 231571/F20) for support. This work has received support from the Norwegian Supercomputing Program (NOTUR) through a grant of computer time (Grant No. NN4654K). We acknowledge computational resources from CSC - the Finnish IT Center for Science as well as the Finnish Grid and Cloud Infrastructure (persistent identifier urn:nbn:fi:research-infras2016072533). We thank Henrik Ottosson for discussion and Maria Dimitrova for discussions of visualization aspects.

\section{References}

1 E. Hückel, Z. Phys., 1931, 70, 204-286.

2 E. Hückel, Z. Phys., 1931, 72, 310-337. 
3 M. Solá, Front. Chem., 2017, 5, 22.

4 J.-Y. Shin, K. S. Kim, M.-C. Yoon, J. M. Lim, Z. S. Yoon, A. Osuka and D. Kim, Chem. Soc. Rev., 2010, 39, 2751-2767.

5 C. Kumar, H. Fliegl and D. Sundholm, J. Phys. Chem. A, 2017, 121, 7282-7289.

6 R. Gershoni-Poranne and A. Stanger, Chem. Soc. Rev., 2015, 44, 6597-6615.

7 J. A. N. F. Gomes and R. B. Mallion, Chem. Rev., 2001, 101, 1349-1384.

8 P. von Ragué Schleyer, C. Maerker, A. Dransfeld, H. Jiao and N. J. R. van Eikema Hommes, J. Am. Chem. Soc., 1996, 118, 6317-6318.

9 R. Herges and D. Geuenich, J. Phys. Chem. B, 2001, 105, 3214-3220.

10 D. Geuenich, K. Hess, F. Köhler and R. Herges, Chem. Rev., 2005, 105, 3758-3772.

11 H. Fliegl, J. Jusélius and D. Sundholm, J. Phys. Chem. A, 2016, 120, 5658-5664.

12 D. Sundholm, R. J. F. Berger and H. Fliegl, Phys. Chem. Chem. Phys., 2016, 18, 15934-15942.

13 S. Van Damme, G. Acke, R. W. A. Havenith and P. Bultinck, Phys. Chem. Chem. Phys., 2016, 18, 11746-11755.

14 M. Dimitrova and D. Sundholm, Phys. Chem. Chem. Phys., 2018, 20, 1337-1346.

15 G. Acke, S. Van Damme, R. W. A. Havenith and P. Bultinck, J. Comput. Chem., 2018, 39, 511-519.

16 P. Lazzeretti, Phys. Chem. Chem. Phys., 2004, 6, 217-223.

17 F. Feixas, E. Matito, J. Poater and M. Solá, J. Comput. Chem., 2008, 29, 1543-1554.

18 F. Feixas, J. O. C. Jiménez-Halla, E. Matito, J. Poater and M. Solá, J. Chem. Theory Comput., 2010, 6, 1118-1130.

19 D. Sundholm, H. Fliegl and R. J. Berger, Wiley Interdiscip. Rev.: Comput. Mol. Sci., 2016, 6, 639-678.

20 H. Fliegl, R. R. Valiev, F. Pichierri and D. Sundholm, Chem. Modell., 2018, 14, 1-42.

21 S. Saito and A. Osuka, Angew. Chem., Int. Ed., 2011, 50, 4342-4373.

22 T. Tanaka and A. Osuka, Chem. Rev., 2017, 117, 2584-2640.

23 S. Hiroto, Y. Miyake and H. Shinokubo, Chem. Rev., 2017, 117, 2910-3043.

24 R. R. Valiev, I. Benkyi, Y. Konyshev, H. Fliegl and D. Sundholm, J. Phys. Chem. A, 2018, 122, 4756-4767.

25 S. Shimizu, N. Aratani and A. Osuka, Chem. - Eur. J., 2006, 12, 4909-4918.

26 A. Osuka and S. Saito, Chem. Commun., 2011, 47, 4330-4339.

27 T. Higashino, J. M. Lim, T. Miura, S. Saito, J.-Y. Shin, D. Kim and A. Osuka, Angew. Chem., Int. Ed., 2010, 49, 4950-4954.

28 T. Higashino and A. Osuka, Chem. Sci., 2013, 4, 1087-1091.

29 Y. M. Sung, M. Yoon, J. M. Lim, H. Rath, K. Naoda, A. Osuka and D. Kim, Nat. Chem., 2015, 7, 418-422.

30 K. Naoda and A. Osuka, Asian J. Org. Chem., 2017, 6, 1205-1208.

31 H. Fliegl, D. Sundholm, S. Taubert and F. Pichierri, J. Phys. Chem. A, 2010, 114, 7153-7161.

32 H. Fliegl, D. Sundholm and F. Pichierri, Phys. Chem. Chem. Phys., 2011, 13, 20659-20665.
33 W.-Y. Cha, T. Kim, A. Ghosh, Z. Zhang, X.-S. Ke, R. Ali, V. M. Lynch, J. Jung, W. Kim, S. Lee, S. Fukuzumi, J. S. Park, J. L. Sessler, T. K. Chandrashekar and D. Kim, Nat. Chem., 2017, 9, 1243-1248. 34 M. Ishida, S.-J. Kim, C. Preihs, K. Ohkubo, J. M. Lim, B. S. Lee, J. S. Park, V. M. Lynch, V. V. Roznyatovskiy, T. Sarma, P. K. Panda, C.-H. Lee, S. Fukuzumi, D. Kim and J. L. Sessler, Nat. Chem., 2013, 5, 15-20.

35 T. Yoshida, W. Zhou, T. Furuyama, D. B. Leznoff and N. Kobayashi, J. Am. Chem. Soc., 2015, 137, 9258-9261.

36 T. Furuyama and N. Kobayashi, Phys. Chem. Chem. Phys., 2017, 19, 15596-15612.

37 D. Shimizu and A. Osuka, Chem. Sci., 2018, 9, 1408-1423.

38 T. Y. Gopalakrishna, J. S. Reddy and V. G. Anand, Angew. Chem., Int. Ed., 2014, 53, 10984-10987.

39 N. C. Baird, J. Am. Chem. Soc., 1972, 94, 4941-4948.

40 K. Jorner, B. O. Jahn, P. Bultinck and H. Ottosson, Chem. Sci., 2018, 9, 3165-3176.

41 H. Ottosson, Nat. Chem., 2012, 4, 969-971.

42 M. Rosenberg, C. Dahlstrand, K. Kilså and H. Ottosson, Chem. Rev., 2014, 114, 5379-5425.

43 R. Ayub, O. El Bakouri, K. Jorner, M. Solá and H. Ottosson, J. Org. Chem., 2017, 82, 6327-6340.

44 J. Oh, Y. M. Sung, H. Mori, S. Park, K. Jorner, H. Ottosson, M. Lim, A. Osuka and D. Kim, Chem, 2017, 3, 1-11.

45 M. J. Goldstein and R. Hoffmann, J. Am. Chem. Soc., 1971, 93, 6193-6204.

46 M. J. Goldstein, Nat. Chem., 1967, 89, 6357-6359.

47 J. B. Grutzner and S. Winstein, J. Am. Chem. Soc., 1970, 92, 3186-3187.

48 J. B. Grutzner and W. L. Jorgensen, J. Am. Chem. Soc., 1981, 103, 1372-1375.

49 D. Geuenich, K. Hess, F. Kohler and R. Herges, Chem. Rev., 2005, 105, 3758-3772.

50 J. Jusélius, D. Sundholm and J. Gauss, J. Chem. Phys., 2004, 121, 3952-3963.

51 S. Taubert, D. Sundholm and J. Jusélius, J. Chem. Phys., 2011, 134, 054123.

52 H. Fliegl, S. Taubert, O. Lehtonen and D. Sundholm, Phys. Chem. Chem. Phys., 2011, 13, 20500-20518.

53 A. D. Becke, J. Chem. Phys., 1993, 98, 5648-5652.

54 C. Lee, W. Yang and R. G. Parr, Phys. Rev. B: Condens. Matter Mater. Phys., 1988, 37, 785-789.

55 A. Schäfer, H. Horn and R. Ahlrichs, J. Chem. Phys., 1992, 97, 2571-2577.

56 R. Ahlrichs, M. Bär, M. Häser, H. Horn and C. Kölmel, Chem. Phys. Lett., 1989, 162, 165-169.

57 F. Furche, R. Ahlrichs, C. Hättig, W. Klopper, M. Sierka and F. Weigend, Wiley Interdiscip. Rev.: Comput. Mol. Sci., 2014, 4, 91-100.

58 Y. Zhao and D. G. Truhlar, Theor. Chem. Acc., 2006, 120, 215-241.

59 A. D. Becke, J. Chem. Phys., 1993, 98, 1372-1377.

60 F. Weigend and R. Ahlrichs, Phys. Chem. Chem. Phys., 2005, 7, 3297-3305.

61 M. Kollwitz, M. Häser and J. Gauss, J. Chem. Phys., 1998, 108, 8295-8301. 
62 K. Reiter, F. Mack and F. Weigend, J. Chem. Theory Comput., 2018, 14, 191-197.

63 M. J. Frisch, G. W. Trucks, H. B. Schlegel, G. E. Scuseria, M. A. Robb, J. R. Cheeseman, V. G. Zakrzewski, J. A. Montgomery Jr, R. E. Stratmann, J. C. Burant, S. Dapprich, J. M. Millam, A. D. Daniels, K. N. Kudin, M. C. Strain, O. Farkas, J. Tomasi, V. Barone, M. Cossi, R. Cammi, B. Mennucci, C. Pomelli, C. Adamo, S. Clifford, J. Ochterski, G. A. Petersson, P. Y. Ayala, Q. Cui, K. Morokuma, D. K. Malick, A. D. Rabuck, K. Raghavachari, J. B. Foresman, J. Cioslowski, J. V. Ortiz, A. G. Baboul, B. B. Stefanov, G. Liu, A. Liashenko, P. Piskorz, I. Komaromi, R. Gomperts, R. L. Martin, D. J. Fox, T. Keith, M. A. Al-Laham, C. Y. Peng, A. Nanayakkara, C. Gonzalez, M. Challacombe, P. M. W. Gill, B. Johnson, W. Chen, M. W. Wong, J. L. Andres, C. Gonzalez, M. HeadGordon, E. S. Replogle and J. A. Pople, Gaussian 09, Revision A.02, Gaussian, Inc., Pittsburgh PA, 2016.

64 R. Ditchfield, Mol. Phys., 1974, 27, 789-807.
65 K. Wolinski, J. F. Hinton and P. Pulay, J. Am. Chem. Soc., 1990, 112, 8251-8260.

66 M. Rauhalahti, S. Taubert, D. Sundholm and V. Liegeois, Phys. Chem. Chem. Phys., 2017, 19, 7124-7131.

67 R. R. Valiev, H. Fliegl and D. Sundholm, Chem. Commun., 2017, 53, 9866-9869.

68 M. Torrent-Sucarrat, S. Navarro, F. P. Cossío, J. M. Anglada and J. M. Luis, J. Comput. Chem., 2017, 38, 2819-2828.

69 J. Ahrens, B. Geveci and C. Law, ParaView: An End-User Tool for Large Data Visualization, Visualization Handbook, Elsevier, 2005, ISBN-13:978-0123875822, see also: http://www. paraview.org.

70 W. Humphrey, A. Dalke and K. Schulten, J. Mol. Graphics, 1996, 14, 33-38.

71 G. A. Zhurko and D. A. Zhurko, http://www.chemcraftprog.com/. 72 A. A. Granovsky, J. Chem. Phys., 2011, 134, 214113.

73 A. A. Granovsky, Firefly, version 8.0.0, http://classic.chem. msu.su/gran/firefly/index.html. 